\title{
Supernumerary teeth vary depending on gender
}

\begin{abstract}
Erika Calvano Küchler(a) Adriana Gomes da Costa(b) Marcelo de Castro Costa(b) Alexandre Rezende Vieira ${ }^{(c)}$ José Mauro Granjeiro(a)
\end{abstract}

\footnotetext{
(a) Biology Institute, Clinical Research Unit, Fluminense Federal University, Niterói, RJ, Brazil.

(b) Department of Pediatric Dentistry and Orthodontics, Federal University of Rio de Janeiro, Rio de Janeiro, RJ, Brazil.

(c) Department of Oral Biology, University of Pittsburgh, Pittsburgh, PA, USA.
}

Corresponding author:

José Mauro Granjeiro

R. Outeiro de São João Batista, s/n -

Centro

Niterói - RJ - Brazil

CEP: 24020-150

E-mail:jmgranjeiro@gmail.com

Received for publication on Aug 04, 2010 Accepted for publication on Nov 26, 2010
Abstract: The presence of supernumerary teeth (ST) is a dental developmental anomaly of patterning and morphogenesis. Its variability of morphology, location and developmental timing can shed light on its etiology. In this work we report ST patterns. Orthopantomograms of 1,166 pediatric subjects were examined and the morphology, location and timing of the formation of ST were determined. The frequency of supernumerary teeth in the studied population was $2.3 \%(\mathrm{n}=27)$. Twenty-five subjects presented one ST. Maxilla midline was the most commonly affected region (nine cases). We noted high incidence of conical morphology in the midline region. Only teeth with tuberculate morphology presented delayed formation. ST in the midline region occurred more often in males whereas ST in the incisor region were more common in females. In conclusion, ST patterns vary depending on gender.

Descriptors: Incidence; Tooth, Unerupted; Tooth, Supernumerary.

\section{Introduction}

Patterning alterations in human dentition often occur and are characterized by alterations in the number, size and shape of teeth. Supernumerary teeth (ST) is a number and morphology developmental alteration and results in the formation of teeth in excess of the usual number. ${ }^{1,2}$ Its prevalence is higher in males than females $(2: 1){ }^{3}$ The reported frequency in permanent dentition varies from $0.1 \%$ to $3.6 \%$ in the general population $^{4-6}$ and a few cases of inherited forms have been reported. ${ }^{7,8}$ ST are commonly found in syndromes ${ }^{1,9}$ and are more common in patients with cleft lip and palate in comparison to the general population. ${ }^{10}$

Classification of ST can be on the basis of morphology and region. Morphology variations include conical types, tuberculate types and supplemental teeth. ${ }^{11}$ They can be found in almost any region of the dental arch but occur more frequently in the maxilla, especially in the anterior segment. ${ }^{6,12}$ ST may occur singularly or in multiple teeth, and unilaterally or bilaterally. ${ }^{11}$

We have proposed that specific dental developmental alterations could serve as clinical markers for the risks of more craniofacial anomalies. ${ }^{10,13,14}$ Although the epidemiology of ST is well established, the exact genetic etiology and molecular mechanism that leads to ST remain largely unclear. Therefore, the aim of this study was to investigate the patterns of ST in a pediatric population from Rio de Janeiro, Brazil. 


\section{Materials and Methods}

Clinical records and orthopantomograms from 1,198 subjects treated at the Rio de Janeiro Federal University Continuing Education Clinical Program in Pediatric Dentistry constituted the sample. The patients' ages varied from 6 to 12 years, and they were treated between January 1999 and November 2007.

All radiographs were examined by the same professional using a protocol previously reported..$^{15}$ The protocol of this study was reviewed and approved by Rio de Janeiro Federal University's institutional review board, and each subject in the project signed an age-appropriate informed consent form, along with his/her parents, who signed a detailed informed consent form.

Cases with poor-quality radiographs were excluded. No cases were found to have an underlying syndrome and/or cleft lip and palate.

\section{Diagnostic criteria of ST}

A supernumerary tooth is one that is additional to the normal series. ST were registered in the permanent dentition and were defined when initial tooth formation was visible in the orthopantomograms. The calcification stage allowed the determination of the timing of the formation in relation to the permanent teeth in the affected region.

The ST morphology classification that we used was previously reported. ${ }^{16}$ Supernumerary teeth are classified as conical (small peg-shaped or conical), tuberculate (with more than one cusp or tubercle frequently described as barrel-shaped and may be invaginated) and supplemental (refers to a duplica-

Table 1 - Relationship between affected region and morphology of supernumerary teeth.

\begin{tabular}{c|c|c|c|c}
\hline \multirow{2}{*}{ Region } & \multicolumn{4}{|c}{ Morphology } \\
\cline { 2 - 5 } & Conical & Supplemental & Tuberculate & Total \\
\hline Midline & 9 & - & - & 9 \\
\hline Incisor & 2 & 4 & 2 & 8 \\
\hline Canine & 3 & 1 & 1 & 5 \\
\hline Premolar & 1 & - & 4 & 5 \\
\hline Total & 15 & 5 & 7 & 27 \\
\hline
\end{tabular}

Note: Supernumerary teeth in the molar region were absent. tion of teeth in the normal series).

\section{Statistical Analyses}

The data were processed and analyzed using the Epi Info3.5.1 statistical software package (Centers for Disease Control and Prevention, Washington, D.C., USA). Odds ratio calculations and chi-square or Fisher's exact tests at a level of significance of 0.05 were used.

\section{Results}

Among the 1,166 radiographs studied, 586 were from females and 580 from males. The age of the population studied ranged from 6 years to 12 years (median age: 8.9 years, standard deviation: 1.7 ).

Twenty-seven subjects presented ST $(2.3 \%), 25$ showing only one ST. Two cases had two ST each. Both cases had tuberculated teeth located bilaterally in the lower premolar region. No subjects presented multiple ST (3 or more ST). ST predominated in the maxilla $(\mathrm{n}=21)$, while only six cases were in the mandible $(\mathrm{p}=0.00004)$.

One-third of our sample showed conical morphology in the midline region (Table 1). Considering the sagittal position of the ST, 13 were in the normal position of eruption and 14 were in an oblique position. None were in the inverted position. Only the tuberculated form presented delayed formation.

No statistically significant differences in the frequency of supernumerary teeth based on gender were found $(\mathrm{p}=0.3)$. Sixteen males and eleven females were affected, for a male-to-female ratio of 1.45:1 (Table 2).

Table 2 - Male-to-female ratio and type of ST.

\begin{tabular}{c|c|c|c}
\hline $\begin{array}{c}\text { Parameter } \\
\text { analyzed of ST }\end{array}$ & Description & $\begin{array}{c}\text { Male:female } \\
\text { ratio }\end{array}$ & p-value \\
\hline \multirow{4}{*}{ Region } & Midiline & $8: 1$ & $0.009^{*}$ \\
\cline { 2 - 4 } & Incisor & $0.3: 1$ & $0.04^{*}$ \\
\cline { 2 - 4 } & Canine & $0.5: 1$ & 0.5 \\
\cline { 2 - 4 } & Premolar & $4: 1$ & $0.05^{*}$ \\
\hline \multirow{4}{*}{ Morphology } & Conical & $2.75: 1$ & $0.01^{*}$ \\
\cline { 2 - 4 } & Supplemental & $0.25: 1$ & $0.05^{*}$ \\
\cline { 2 - 4 } & Tuberculate & $1.3: 1$ & 0.5 \\
\hline
\end{tabular}

*Statistically significant differences. 


\section{Discussion}

ST is a unique developmental dental anomaly of patterning and morphogenesis. ${ }^{1}$ The variability of the phenotype (i.e., tooth morphology, location in the dental arch and developmental timing of ST) raises interesting questions about molecular and human genetics aspects. In this work we studied a sample of pediatric subjects and investigated specific patterns of ST that could be used to study the etiology of this condition.

In the general population the reported frequency of ST depends on the group studied. The group evaluated in our study is an admixed population from Rio de Janeiro, which is comprised of subjects of various mixtures of European and African descent with a very small percentage of indigenous South American. Here we found a frequency of ST of $2.3 \%$, similar to that of previous reports. ${ }^{4-6} \mathrm{We}$ did not find subjects with multiple supernumerary teeth, and indeed this is rare in the general population, representing less than $1 \%$ of the total cases. ${ }^{17}$ Multiple ST is more commonly associated with cleidocranial dysplasia, Gardner syndrome and cleft lip and palate. ${ }^{7,17}$

The conical shape was the most frequently observed morphology in our study, followed by the tuberculate and supplemental shapes, which corroborates previous findings. ${ }^{18}$ Our study also agrees with the majority of the reports that describe maxilla midline as the most common pattern, and the incidence of mesiodens in other studies varied from 0.15 to $3.8 \%{ }^{19}$

However, the stage of ST development has not been well described. Previous reports have suggested that the developmental stage of ST is directly linked to morphology, since fully developed ST were commonly in conical and supplemental types. ${ }^{18}$ In our study, the tuberculate morphology was also associated with delayed tooth formation.

The etiology of ST is largely unclear, and the different morphologies, affected regions and developmental timing of ST support various theories about this condition. One theory suggests that the ST is created as a result of a dichotomy of the tooth bud. Another theory, well supported in the literature, is the hyperactivity theory, which suggests that ST are formed as a result of local, independent, conditioned hyperactivity of the dental lamina. ${ }^{20}$ It has also been suggested that they are a result of atavism, meaning a reversion to a more primitive type of dentition. However, atavism cannot explain the presence of ST in the canine and midline regions or tuberculate forms in any region. On the other hand, supplemental forms may be a result of atavism. Heredity may also play a role in the occurrence of this anomaly, as ST are more common in the relatives of affected children than in the general population ${ }^{16}$ and mutant genes can also account for this anomaly. It is difficult to establish a clear etiology for ST and different forms of ST are not easily explained.

Dental anomalies are a multifactorial condition and present gender prevalence discrepancies: while ST are more common in males, tooth agenesis is more common in females. We have previously reported the sexual dimorphism in subphenotypes of tooth agenesis. ${ }^{13}$ In this study we noted that subphenotypes of ST also presented gender preference: midline and premolar regions were more affected in males, while incisor and canine regions were more affected in females.

The different patterns of clinical presentation of ST may have independent developmental mechanisms and different genetic factors. A better understanding of the clinical presentation of dental anomalies is important to guide molecular studies regarding the etiology of ST. Further investigations with larger sample sizes are needed to allow determination of subphenotypes of ST.

\section{Conclusion}

The midline conical ST is the most common type of ST. The incidence of ST and their location and morphology vary depending on gender.

\section{Acknowledgements}

We thank Melissa Carp for revising the manuscript text for grammar and style. Support for this work was provided by CAPES (ECK), by FAPERJ (MCC, JMG) and CNPq (JMG). 


\section{References}

1. D'Souza RN, Klein OD. Unraveling the molecular mechanisms that lead to supernumerary teeth in mice and men: current concepts and novel approaches. Cells Tissues Organs. 2007 Mar;186(1):60-9.

2. Srivatsan P, Aravindha Babu N. Mesiodens with an unusual morphology and multiple impacted supernumerary teeth in a non-syndromic patient. Indian J Dent Res. 2007 JulSep;18(3):138-40.

3. Sharma A. Familial occurence of mesiodens--a case report. J Indian Soc Pedod Prev Dent. 2003 Jun;21(2):84-5.

4. Acikgoz A, Acikgoz G, Tunga U, Otan F. Characteristics and prevalence of non-syndrome multiple supernumerary teeth: a retrospective study. Dentomaxillofac Radiol. 2006 May;35(3):185-90.

5. Arathi R, Ashwini R. Supernumerary teeth: a case report. J Indian Soc Pedod Prev Dent. 2005 Jun;23(2):103-5.

6. Zhu JF, Marcushamer M, King DL, Henry RJ. Supernumerary and congenitally absent teeth: a literature review. J Clin Pediatr Dent. 1996 Winter;20(2):87-95.

7. Yusof WZ, Awang MN. Multiple impacted supernumerary teeth. Oral Surg Oral Med Oral Pathol. 1990 Jul;70(1):126.

8. Burzynski NJ, Escobar V. Classification and genetics of numeric anomalies of dentition. Birth Defects Orig Artic Ser. 1983 May;19(1):95-106.

9. Acerbi AG, de Freitas C, de Magalhaes MH. Prevalence of numeric anomalies in the permanent dentition of patients with Down syndrome. Spec Care Dentist. 2001 MarApr;21(2):75-8.

10. Letra A, Menezes R, Granjeiro JM, Vieira AR. Defining subphenotypes for oral clefts based on dental development. J Dent Res. 2007 Oct;86(10):986-91.
11. Scheiner MA, Sampson WJ. Supernumerary teeth: a review of the literature and four case reports. Aust Dent J. 1997 Jun;42(3):160-5.

12. Giancotti A, Grazzini F, De Dominicis F, Romanini G, Arcuri C. Multidisciplinary evaluation and clinical management of mesiodens. J Clin Pediatr Dent. 2002 Spring;26(3):233-7.

13. Küchler EC, Risso PA, Costa MD, Modesto A, Vieira AR. Studies of dental anomalies in a large group of school children. Arch Oral Biol. 2008 May;53(10):941-6

14. Menezes R, Vieira AR. Dental anomalies as part of the cleft spectrum. Cleft Palate Craniofac J. 2008 Jul;45(4):414-9.

15. Küchler E, Risso P, Costa M, Modesto A, Vieira AR. Assessing the proposed association between tooth agenesis and taurodontism in 975 paediatric subjects. Int J Paediatr Dent. 2008 May;18(3):231-4.

16. Garvey MT, Barry HJ, Blake M. Supernumerary teeth--an overview of classification, diagnosis and management. J Can Dent Assoc. 1999 Dec;65(11):612-6.

17. Vucicevic Boras V, Mohamad Zaini Z, Savage NW. Supernumerary tooth with associated dentigerous cyst in an infant. A case report and review of differential diagnosis. Aust Dent J. 2007 Jun;52(2):150-3.

18. De Oliveira Gomes C, Drummond SN, Jham BC, Abdo EN, Mesquita RA. A survey of 460 supernumerary teeth in Brazilian children and adolescents. Int J Paediatr Dent. 2008 Mar;18(2):98-106.

19. Prabhu NT, Rebecca J, Munshi AK. Mesiodens in the primary dentition--a case report. J Indian Soc Pedod Prev Dent. 1998 Sep;16(3):93-5.

20. Liu JF. Characteristics of premaxillary supernumerary teeth: a survey of 112 cases. ASDC J Dent Child. 1995 JulAug;62(4):262-5. 